\title{
Experimental and Theoretical In Situ Spectral Magneto- Ellipsometry Study of Layered Ferromagnetic Structures
}

\author{
O. A. Maximova ${ }^{a, b}, *$, S. A. Lyaschenko ${ }^{a}$, M. A. Vysotin ${ }^{a, b}$, I. A. Tarasov ${ }^{a}$, I. A. Yakovlev ${ }^{a}$, \\ D. V. Shevtsov ${ }^{a}$, A. S. Fedorov ${ }^{a, b}$, S. N. Varnakov ${ }^{a}$, and S. G. Ovchinnikov ${ }^{a, b}$ \\ ${ }^{a}$ Kirensky Institute of Physics, Federal Research Center KSC, Siberian Branch, Russian Academy of Sciences, \\ Akademgorodok, Krasnoyarsk, 660036 Russia \\ ${ }^{b}$ Siberian Federal University, Krasnoyarsk, 660041 Russia \\ *e-mail: maximo.a@mail.ru \\ Received February 27, 2019; revised June 14, 2019; accepted June 14, 2019
}

\begin{abstract}
A method for processing of in situ spectral magneto-ellipsometry data has been developed to analyze planar ferromagnetic nanostructures. A multilayer model containing a ferromagnetic layer with two interfaces, a nonferromagnetic buffer layer, and a nonferromagnetic substrate has been tested within a new approach to the interpretation of magnetic-field-modulated spectral ellipsometric measurements involving the magnetooptical Kerr effect in the transverse configuration. In particular, the effect of the thickness of the ferromagnetic layer on the results of magneto-ellipsometric measurements has been analyzed. The measurements have been performed with polycrystalline Fe films with different thicknesses on a nonferromagnetic $\mathrm{SiO}_{2} / \mathrm{Si}(100)$ surface. The diagonal and off-diagonal components of the complex dielectric tensor in the spectral range of $1.38-3.45 \mathrm{eV}$ have been determined by processing spectral magneto-ellipsometric data. The results have been compared to the available data obtained by other authors and to the calculation of the dielectric tensor of Fe within the density functional theory.
\end{abstract}

DOI: $10.1134 / \mathrm{S} 0021364019150098$

1. The development of tools for nondestructive in situ control of planar ferromagnetic nanostructures is important for ensuring the reproducible synthesis of such nanostructures and a highly accurate control of their physical properties. Ellipsometric measurements of ferromagnetic materials in an external magnetic field allow determining both diagonal and off-diagonal components of the complex dielectric tensor, as well as the magnetic circular dichroism [1]. Approaches to the application of magneto-ellipsometry to determine the complete dielectric tensor have already been developed. In particular, the authors of [2] proposed an approach to the calculation of the offdiagonal components of the dielectric tensor of a multilayer ferromagnetic structure based on the Mueller matrices, in particular, for optically anisotropic media, using normally incident light for the polar Kerr effect. Further, successful measurements were performed using the method proposed in [2] to analyze an opaque ferromagnetic film at different temperatures [3]. However, the model of a semi-infinite ferromagnetic layer developed for bulk materials and thick films is insufficient for interpreting experimental data for ferromagnetic layers with thicknesses comparable with the thickness of the skin layer in the used spectral range. The authors of [4] developed a method of generalized magneto-optical spectral ellipsometry involv- ing an octupole electromagnet and measured the complete dielectric tensor of a transparent isotropic cobalt film with a surface oxide layer. However, only a few applications of magneto-ellipsometry for the in situ analysis of a multilayer ferromagnetic medium were reported. This is possibly because the Mueller matrix technique is cumbersome in application to the analysis of the multilayer ferromagnetic structure in combination with construction constraints for the introduction of a multipole electromagnet directly into the synthesis chamber.

The aims of this work are, first, to test our approach to the analysis of magneto-ellipsometric data, which does not require fourth order Mueller matrices and is based on ellipsometric relations where the magnetooptical contribution is taken into account as a perturbation, and, second, to perform in situ analysis of a simple dipole electromagnetic system based on the transverse magneto-optical Kerr effect [5]. We emphasize that all measurements were performed in situ and the sample is not exposed to the atmosphere; for this reason, the surface is free from additional oxide and other layers that would be necessary to take into account in the analysis. The processing of experimental data includes (i) the separation of optical characteristics of the medium from the ellipsometric parameters measured without magnetic field, (ii) the 
separation of the magneto-optical contribution in the ellipsometric relations by analyzing measurements of complex reflection coefficients for the $p$ - and $s$-polarized incident light at the remagnetization of the sample, (iii) the choice of a reflecting medium model to describe the sample, and (iv) the calculation of all components of the dielectric tensor using optical and magneto-optical data. Thus, it is sufficient to perform measurements of ellipsometric angles in a given spectral range both under the application of the magnetic field and in the demagnetized state without the necessity of variation of the position of the sample and additional measuring instruments.

In this work, we analyze data that were obtained in the experiment on ferromagnetic $\mathrm{Si}$ (substrate) $-\mathrm{SiO}_{2}$ (buffer layer)-Fe(ferromagnetic layer) nanostructures with different thicknesses of the Fe layer and were processed using the multilayer model of reflecting systems that contains ferromagnetic and nonferromagnetic films on a semi-infinite nonferromagnetic substrate [6] and takes into account the $\mathrm{SiO}_{2} / \mathrm{Fe}$ and $\mathrm{Fe} /$ vacuum interfaces. The listed materials were chosen because numerous optical and magneto-optical measurements for them are available for quantitative comparison with our measurements and calculations $[3,7,8]$.

In addition, it is relevant to apply the developed original approach to calculate the complete dielectric tensor of polycrystalline isotropic ferromagnetic films with different thicknesses using in situ measurement data. It is technologically and methodically difficult to obtain this information on the ferromagnetic structure in the in situ regime. The components of the dielectric tensor thus determined from the analysis of experimental data are compared to the ab initio density functional theory (DFT) calculation.

2. Three experimental samples were prepared by the method of thermal evaporation of high-purity $(99.99+\%)$ iron and its deposition in an ultrahigh vacuum of $10^{-7} \mathrm{~Pa}$ on a $300-\mu \mathrm{m}$-thick boron-doped single-crystal silicon substrate with the (100) crystallographic surface orientation. $\mathrm{A} \mathrm{SiO}_{2}$ layer thinner than $2 \mathrm{~nm}$ was preliminarily formed chemically on the substrate surface using the method proposed in [9]. The thickness of the formed silicon dioxide layer was measured by the spectral ellipsometry method in air and was further used to adjust the optical circuit for in situ spectral ellipsometric measurements, in particular, to determine the angle of incidence of the probe beam on the sample using the simple single-layer model with the known dispersions of optical constants of the $\mathrm{Si}$ substrate and $\mathrm{SiO}_{2}$ layer. Before the deposition of iron, the $\mathrm{SiO}_{2} / \mathrm{Si}(100)$ substrates were annealed in ultrahigh vacuum to $670 \mathrm{~K}$ by means of the direct flow of current for degassing and cleaning of the surface. In situ spectral ellipsometric measurements after annealing show a decrease in the thickness of the $\mathrm{SiO}_{2}$ layer by $0.6 \mathrm{~nm}$ on average, which is assumingly due to close disper- sions of the optical constants of water and silicon dioxide at subnanometer thicknesses and to the annealinginduced desorption of water from the substrate surface.

The evaporation of iron in ultrahigh vacuum was performed by the autocrucible method with electronbeam heating. The technological conditions of $\mathrm{Fe}$ deposition and the parameters of the substrates are identical for all three samples and differ only in the $\mathrm{Fe}$ deposition time. The deposition of $\mathrm{Fe}$ and subsequent in situ measurements were performed at room temperature with a special sample holder [10]. The Fe flow velocity was preliminarily calibrated using X-ray spectral fluorescence analysis with an ARL QUANT'X (Thermo Scientific) analyzer and was about $1.3 \mathrm{~nm} / \mathrm{min}$. To obtain additional information on the thickness and chemical composition of the $\mathrm{Fe}$ films, all samples were then studied by the X-ray spectral fluorescence method with the recalculation to an iron density of $7.874 \mathrm{~g} / \mathrm{cm}^{3}$ under normal conditions. According to X-ray spectral fluorescence data, the thicknesses of the Fe layers in samples 1, 2, and 3 were $(77.0 \pm 0.6) \mathrm{nm},(33.5 \pm 0.6) \mathrm{nm}$, and $(11.5 \pm 0.6) \mathrm{nm}$, respectively. According to the spectral ellipsometry data, the thicknesses of the $\mathrm{SiO}_{2}$ layers in samples 1, 2, and 3 were $(0.7 \pm 0.2) \mathrm{nm},(1.8 \pm 0.2) \mathrm{nm}$, and $(1.0 \pm$ $0.2) \mathrm{nm}$.

Taking into account that real multilayer nanostructures do not have mathematically smooth interfaces, we analyzed the effect of two additional mixed layers (Fig. 1) identical for all samples: (i) a 0.12-nm layer with an iron content of $50 \mathrm{vol} \%$ at the $\mathrm{Fe} / \mathrm{SiO}_{2}$ interface and (ii) a $0.58-\mathrm{nm}$ layer with an iron content of 50 vol $\%$ at the $\mathrm{Fe} /$ vacuum interface. The thicknesses of the mixed layers were chosen according to the rms roughness obtained from atomic force microscopy data and correspond to the data for similar structures from [11]. An Fe content of $50 \mathrm{vol} \%$ constant over the depth was chosen to simplify the calculation of the mixed layer model. In addition, the magneto-optical contribution from both transition layers was disregarded in this work. The reason is that, according to our additional experiments, this contribution for in situ magneto-ellipsometric measurements at averaged thicknesses of the polycrystalline Fe layer on $\mathrm{SiO}_{2}$ less than $6 \mathrm{~nm}$ becomes comparable with the rms error of ellipsometric measurements. Magneto-optical measurements for much thinner ferromagnetic films were also reported (see, e.g., [12], where ex situ measurements were reported). However, in this work, we performed in situ spectral magneto-ellipsometric measurements with a monochromator and a high-pressure xenon arc lamp as a light source, which can technically ensure the average brightness of the probe beam no more than 0.01 of the laser brightness. In view of the additional reflection of the beam from the optical windows of the technological synthesis chamber and a large optical path from the sample to a photodetector 
compared to a table configuration, the resulting intensity of the beam can be three orders of magnitude lower than that of a laser magnetic ellipsometer even at a wavelength of about $500 \mathrm{~nm}$ corresponding to the maximum intensity of the luminescence of the xenon lamp. A reasonable solution could be to increase the signal collection time, but the probability of adsorption of the residual atmosphere on the sample surface with the formation of an oxide film increases in this case. For this reason, in our calculations, we took into account transition mixed layers thinner than $0.6 \mathrm{~nm}$, but we cannot treat them as magnetic.

Features of the electronic structure at the mathematical boundaries of the mixed layers and boundary materials are disregarded in this work because the variation of the concentration over the thickness is assumed to be smooth and the materials of the layers have a common origin.

Magneto-ellipsometric measurements included the following stages. Firstly, a magnetic field sufficient for the ferromagnetic saturation of the film was induced on the sample by an electromagnet. Secondly, spectral ellipsometric measurements of the angles $\Psi$ and $\Delta$ in four optical bands [13] with averaging were performed. Thirdly, the remagnetization of the sample with saturation was performed by the application of the magnetic field with the same amplitude but opposite direction. Fourthly, spectral ellipsometric measurements in four optical bands [13] with averaging were again performed. Finally, the magnetic contributions to the measured angles were calculated as the differences $\delta \Psi=\Psi(H)-\Psi(-H)$ and $\delta \Delta=\Delta(H)-\Delta(-H)$ for two magnetic states of the sample. We also calculated the arithmetic mean of the measured angles $\Psi$ and $\Delta$. The described measurement algorithm was repeated 50 times for averaging of data and calculation of the rms error of measurements.

As known, the dielectric tensor of the magnetized isotropic ferromagnetic metal is constructed in terms of induced anisotropy and has the form [14]

$$
[\varepsilon]=\left[\begin{array}{ccc}
\varepsilon_{11} & \varepsilon_{12} & 0 \\
\varepsilon_{21} & \varepsilon_{22} & 0 \\
0 & 0 & \varepsilon_{33}
\end{array}\right]=\left[\begin{array}{ccc}
\varepsilon_{11} & \varepsilon_{12} & 0 \\
-\varepsilon_{12} & \varepsilon_{11} & 0 \\
0 & 0 & \varepsilon_{11}
\end{array}\right] .
$$

Each component of this tensor is a complex number, diagonal components are responsible for the optical properties of the medium, and the off-diagonal components phenomenologically couple magnetism and optics and vanish at zero magnetization. Information on the electronic structure of the sample can be obtained with knowledge of all components of the dielectric tensor. The experimental error of the complex dielectric tensor was calculated as the error of indirect measurements from the standard deviation for the sample [15].

3. In situ spectral ellipsometric and magneto-ellipsometric measurements were performed immediately

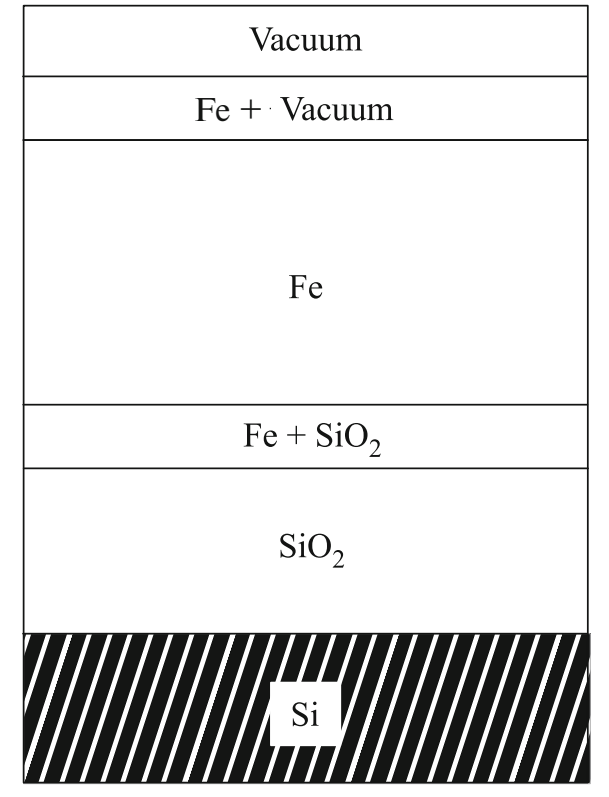

Fig. 1. Schematic of the model used for the experimental sample.

after the synthesis of $\mathrm{Fe} / \mathrm{SiO}_{2} / \mathrm{Si}(100)$ structures in an ultrahigh vacuum chamber. An Ellipse-1891 high speed spectral ellipsometer with an attachment to specify the magnetic field on the sample was applied [16]. According to the preliminary measurements of the remagnetization loop, the ferromagnetic saturation field of the sample was specified at $\pm 2 \mathrm{kOe}$.

Using the ellipsometry data, the spectral dependences of the complex dielectric constant $\varepsilon_{11}$ of $\mathrm{Fe}$ were calculated for all samples by a numerical method within a multilayer model including mixed layers at the interfaces (Fig. 1). The Bruggeman model [17] was used to calculate mixed layers. The optical parameters were calculated by the Spektroskan (Version 1.10) program using the spectral dependence of $\varepsilon_{11}$ for $\mathrm{SiO}_{2}$ from [18]. In turn, the dielectric permittivity of silicon was obtained from the ellipsometric measurements with the $\mathrm{Si}(100)$ single-crystal substrate at room temperature in the spectral range from 1.38 to $3.45 \mathrm{eV}$ taking into account the roughness of the oxide layer by the method from [10]. The possibility of using data on the spectral dependence of $\varepsilon_{11}$ for bulk $\mathrm{SiO}_{2}$ from [18] was shown in [11], where the thickness of the silicon oxide layer was determined from the ellipsometric measurements of $\mathrm{SiO}_{2} / \mathrm{Si}(100)$ films involving bulk data for $\varepsilon_{11}$; the result coincided with the direct electron microscopy measurements. The total thickness of the Fe layer in the calculations of $\varepsilon_{11}$ corresponded to $\mathrm{X}$-ray spectral fluorescence data.

The $\varepsilon_{11}$ values of the Fe layer for all three samples calculated from ellipsometric measurements in the spectral range from 1.38 to $3.45 \mathrm{eV}$ are shown in Fig. 2 

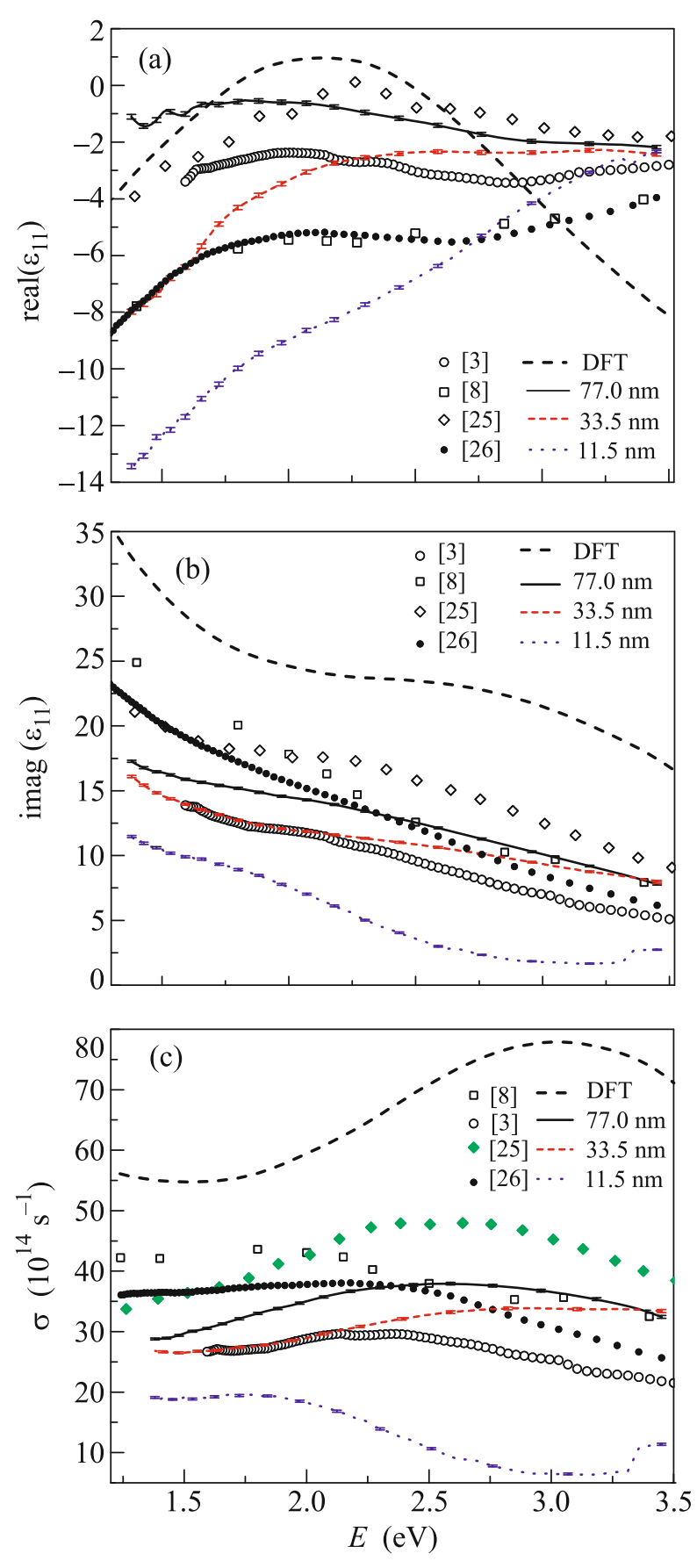

Fig. 2. (Color online) Dispersions of the (a) real and (b) imaginary parts of $\varepsilon_{11}$ and (c) optical conductivity of Fe for three samples with different thicknesses of the layer as calculated from the in situ spectral ellipsometry data and within the DFT compared to the data from $[8,26]$ for bulk samples, from [3] for a 60-nm Fe film, and from [25] (average value for films with thicknesses from 29 to $38 \mathrm{~nm})$.

together with the theoretical calculations and measurements by other authors. The dielectric tensor of Fe was calculated within the DFT [19] implemented in the VASP program packet [20] in the generalized gra- dient approximation [21] of the projected augmented wave method [22] and including the spin-orbit interaction. The plane wave basis was limited by a cutoff energy of $520 \mathrm{eV}$. An $18 \times 18 \times 18$ Monkhorst-Pack mesh [23] was used for the sampling of the Brillouin zone. The diagonal and off-diagonal components of the dielectric tensor were calculated in the random phase approximation disregarding local field effects [24]. Summation involved only vertical interband transitions and the broadening parameter was $0.3 \mathrm{eV}$. The theoretical calculation was performed for the bulk medium disregarding temperature effects.

Calculations of $\varepsilon_{11}$ are in qualitative agreement with measurements by other authors. A monotonic decrease in the imaginary part of $\varepsilon_{11}$ with increasing radiation energy is seen. The components of $\varepsilon_{11}$ depend on the thickness of the iron layer. In particular, as the thickness of the layer decreases, the shape of the curves for the imaginary part of $\varepsilon_{11}$ changes within the entire spectral range. All curves demonstrate a broad maximum whose position depends on the thickness of the Fe layer. To demonstrate the variation of the position of this maximum, we plotted the spectra of the optical conductivity $\left(\sigma=\operatorname{Im}\left(\varepsilon_{11}\right) \omega / 4 \pi\right)$ : a broad maximum near $3 \mathrm{eV}$ for the 33.5-nm sample and the DFT calculation is shifted to 2.6 and $1.8 \mathrm{eV}$ for measurements reported in [25] and the 11.5-nm sample, respectively. A weak broad maximum is observed in the range of 2.3-2.8 eV for the 77.0-nm Fe layer. According to data from $[3,8,26]$, the position of the maximum also depends on the thickness of the $\mathrm{Fe}$ layer: as the thickness increases, the maximum is shifted toward long wavelengths.

To reveal reasons for the maximum on the DFT curve of the optical conductivity near $3 \mathrm{eV}$, we performed the spin-polarized calculation of the electron density of states (DOS) for bulk samples. Figure 3 shows the total DOS and the partial contributions to the DOS from $p$ and $d$ orbitals with spin up and down. The final states of possible electronic transitions can be the $d_{\downarrow}\left(x^{2}-y^{2}\right)$ peak at $+1.92 \mathrm{eV}$ above the Fermi level and the wide $d_{\downarrow}\left(z^{2}\right)$ peak at $+1.5 \mathrm{eV}$. The initial states of the transitions are assumingly the plateau of $d_{\downarrow}(x y)+d_{\downarrow}(y z)+d_{\downarrow}(x z)$ states in the range of $(-1.0$, $-0.5)$ eV below the Fermi level and the peak of $p$ states (with low density compared to $d$ electrons) at $-1.06 \mathrm{eV}$. This gives a broad peak of spin-down electron transitions in the range of $2.5-3.5 \mathrm{eV}$. The entire electronic structure and a broad peak of the optical conductivity $\sigma(\sim 3 \mathrm{eV})$ are in good agreement with DFT calculations in the local spin density approximation, e.g., in [27]. The authors of [27] concluded that the contribution of interband transitions prevails at frequencies above $0.5 \mathrm{eV}$ and that the peak of the imaginary part of the dielectric tensor component $\varepsilon_{11}$ near $2.7 \mathrm{eV}$ is due to spin-down states. However, any 


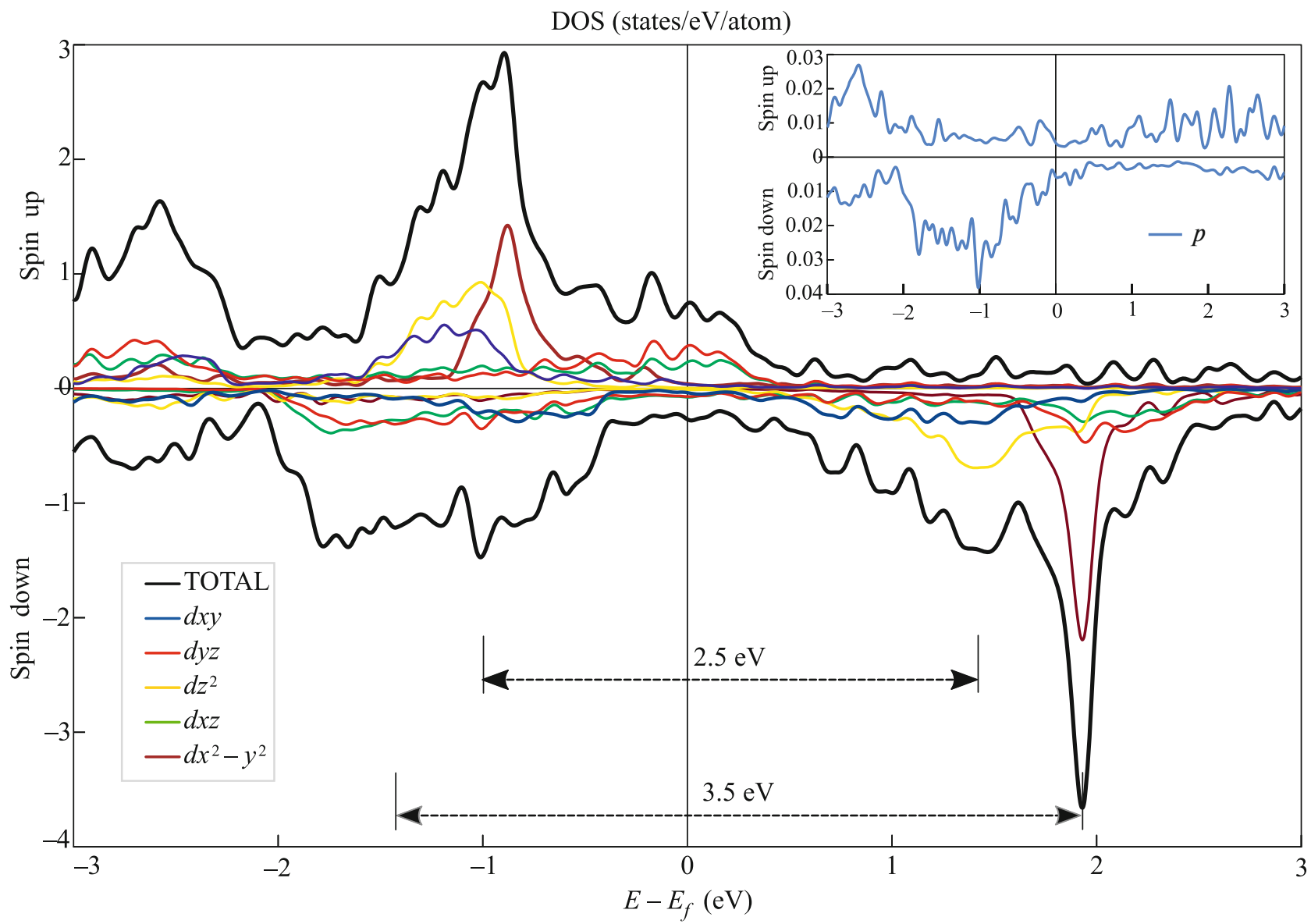

Fig. 3. (Color online) Total electron density of states (DOS) and partial contributions from the $d$ and (inset) $p$ orbitals calculated within the DFT. The energy is measured from the Fermi level.

detailed analysis of $p$ and $d$ states in the region of transitions was not given in [27].

The analysis of the $\mathrm{SiO}_{2}-\mathrm{SiO}_{2} / \mathrm{Fe}-\mathrm{Fe}-\mathrm{Fe} / \mathrm{vac}-$ uum four-layer model on the $\mathrm{Si}$ substrate with the variation of the thicknesses of the $\mathrm{SiO}_{2} / \mathrm{Fe}$ and $\mathrm{Fe} /$ vacuum interface layers from 0 to $1 \mathrm{~nm}$ reveals that the contributions of the interfaces to the ellipsometric angles $\Psi$ and $\Delta$ even for the thinnest 11.5-nm iron layer do not exceed the error of measurements of the angles $\Psi$ and $\Delta$. Thus, if the contribution of interfaces simulating microroughness results in the energy shift of interband transition peaks owing to the contribution of surface plasmons [28], the broadening of the peak of interband transitions in the range of $2.7-3 \mathrm{eV}$ in the DFT calculation toward low energies at a decrease in the thickness of the Fe layer should be expected. However, experimental data demonstrate a more complex picture, where spectrograms of imag $\left(\varepsilon_{11}\right)$ obtained by different authors have peaks with different intensities in different spectral ranges almost independently of the thickness of the Fe layer. Such a picture is also observed for our samples and for other previously published data. The reasons for differences in experimen- tal spectra obtained by other authors can vary from the film deposition rate and the composition of the residual atmosphere in the growing chamber to the nature of an abrasive material at the polishing of bulk samples in early works $[8,26]$.

Figure 4 shows the dispersion dependences of $\varepsilon_{12}$ calculated for a continuous ferromagnetic $\mathrm{Fe}$ layer in all three samples within the multilayer model including the mixed layers at the interfaces (Fig. 1) using magneto-ellipsometric measurement data, as well as the theoretical calculation and experimental data obtained by other authors. The initial calculations were performed within a simpler two-layer model disregarding the mixed layers. However, the comparison of calculations within these two models indicates that the difference between the off-diagonal components $\varepsilon_{12}$ is the most pronounced in the long-wavelength range and increases with a decrease in the thickness of the iron layer. The diagonal components $\varepsilon_{11}$ are the same within the experimental error for any thickness of the iron layer. We choose the multilayer model including the mixed layers because it describes more appropriately the real structure of the samples. 

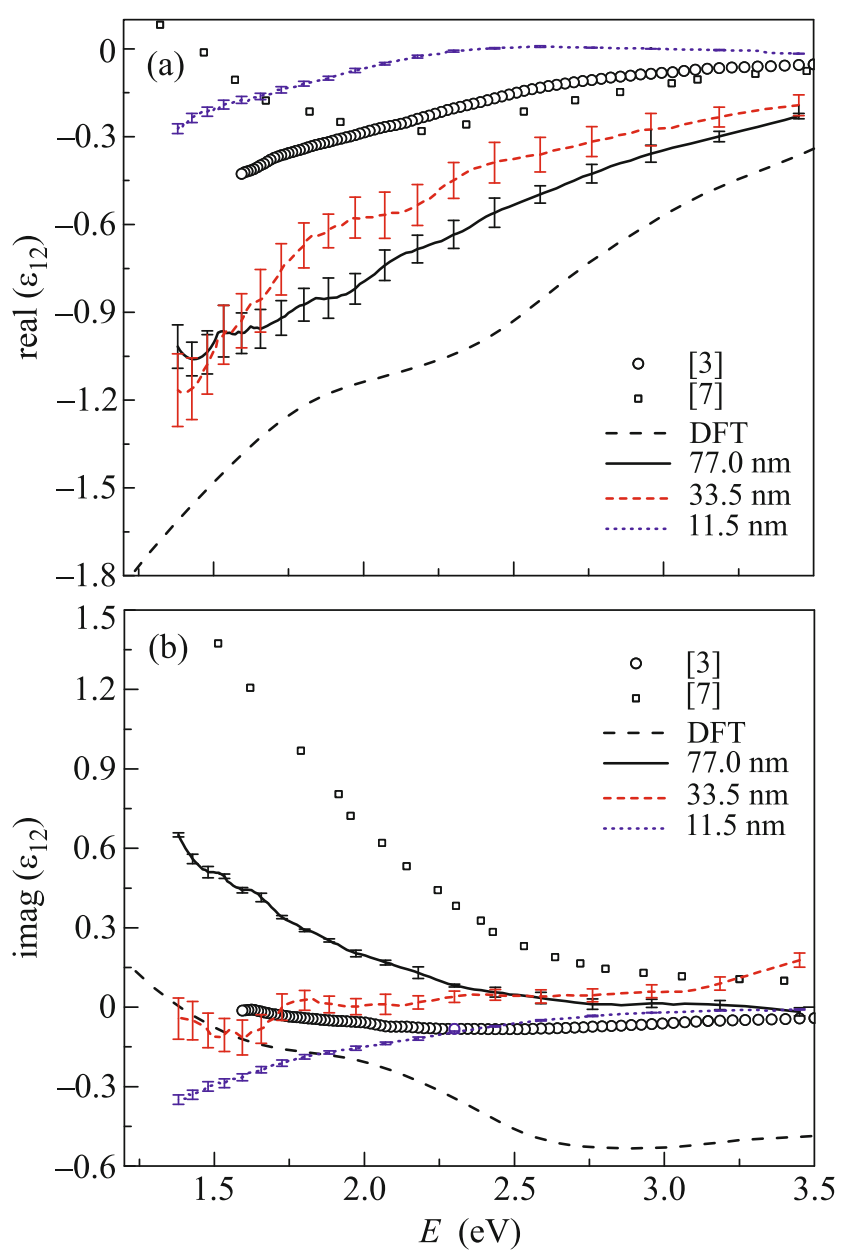

Fig. 4. (Color online) Dispersions of the real and imaginary parts of $\varepsilon_{12}$ for $\mathrm{Fe}$ for three samples with different thicknesses of the layer as calculated from the in situ spectral ellipsometry and within the DFT compared to the data from [7] for bulk samples and from [3] for a 60-nm Fe film.

The comparison of curves shown in Fig. 4 shows qualitative agreement of the DFT calculation for $\varepsilon_{12}$ with the experiment for the 77-nm sample. The characteristic slope of the curve for $\operatorname{imag}\left(\varepsilon_{12}\right)$ of the thick Fe layer coincides with the literature data and the DFT calculation. The slope is also observed in most data of other authors using thick opaque polycrystalline iron films. The amplitude of $\varepsilon_{12}$ generally depends on the thickness of the iron layer and agreement with the theoretical calculation is not observed for thinner films. The experimental results obtained in this work demonstrate change in the slope of $\operatorname{imag}\left(\varepsilon_{12}\right)$ curves and the transition to the negative region with a decrease in the thickness of the Fe layer. The spectrograms of $\varepsilon_{12}$ for the 77-nm Fe film are in agreement with the data obtained in [3]. The spectra for a thick film possibly contain a Drude part from the contribution of free electrons. In this case, a decrease in the
Drude peak can be attributed to a decrease in the contribution of free electrons in polycrystalline thin films because intercrystallite defects and the surface roughness play a noticeable role in real polycrystalline structures, which most probably gives a different spectral response for $\operatorname{imag}\left(\varepsilon_{12}\right)$ of the $11.5-\mathrm{nm}$ film. Furthermore, it is noteworthy that discrepancies with data of other authors are additionally due to iron oxides on the surface of their films. These oxides appear because the measurements were performed after aging of the samples in air, which leads to the formation of an iron oxide layer with a thickness of at least 3-4 nm, which is individual and unknown for an individual experiment. On the contrary, our study was completely performed inside the ultrahigh vacuum chamber immediately after the synthesis of the samples.

4. The performed measurements for thin polycrystalline Fe layers have revealed a strong effect of the thickness on both diagonal and off-diagonal components of the dielectric tensor $\varepsilon$. This effect is most likely due to an increase in the fraction of surface atoms of the polycrystalline layer with a decrease in its thickness and inhomogeneities in the distribution of the magnetic moment. Moreover, the variation of the thickness of the ferromagnetic layer changes not only the amplitude of the components of $\varepsilon$ but also the character of the spectral dependences, which indirectly indicates the energy shift of peaks of characteristic electronic transitions. This shift is confirmed by the DFT calculation of the dielectric tensor $\varepsilon$ for bulk bcc iron, which is in agreement with the measurements for the 77-nm Fe sample.

\section{FUNDING}

This work was supported by the Ministry of Education and Science of the Russian Federation and the Siberian Branch, Russian Academy of Sciences (project nos. 03562018-0061 and II.8.70).

\section{REFERENCES}

1. P. Q. J. Nederpel and J. W. D. Martens, Rev. Sci. Instrum. 56, 687 (1985).

2. Š. Višňovský, K. Postava, and T. Yamaguchi, Czechosl. J. Phys. 51, 917 (2001).

3. G. Neuber, R. Rauer, J. Kunze, T. Korn, C. Pels, G. Meier, U. Merkt, J. Bäckström, and M. Rübhausen, Appl. Phys. Lett. 83, 4509 (2003).

4. K. Mok, N. Du, and H. Schmidt, Rev. Sci. Instrum. 82, 033112 (2011).

5. O. A. Maximova, N. N. Kosyrev, S. N. Varnakov, S. A. Lyashchenko, and S. G. Ovchinnikov, J. Struct. Chem. 55, 1134 (2014).

6. O. A. Maximova, S. G. Ovchinnikov, N. N. Kosyrev, and S. A. Lyaschenko, SibFU J. Math. Phys. 10, 223 (2017).

7. G. S. Krinchik and V. A. Artem'ev, Sov. Phys. JETP 26, 1080 (1968). 
8. G. S. Krinchik, Izv. Akad. Nauk SSSR 28, 481 (1964).

9. I. A. Yakovlev, S. N. Varnakov, B. A. Belyaev, S. M. Zharkov, M. S. Molokeev, I. A. Tarasov, and S. G. Ovchinnikov, JETP Lett. 99, 527 (2014).

10. D. V. Shevtsov, S. A. Lyaschenko, and S. N. Varnakov, Instrum. Exp. Tech. 60, 759 (2017).

11. N. V. Volkov, A. S. Tarasov, E. V. Eremin, S. N. Varnakov, S. G. Ovchinnikov, and S. M. Zharkov, J. Appl. Phys. 109, 123924 (2011).

12. M. Talmadge, J. Gao, M. P. Riley, R. J. Roth, S.-O. Kim, and J. G. Eden, Appl. Phys. Lett. 84, 4197 (2004).

13. S. A. Lyashchenko, I. A. Tarasov, S. N. Varnakov, D. V. Shevtsov, V. A. Shvets, V. N. Zabluda, S. G. Ovchinnikov, N. N. Kosyrev, G. V. Bondarenko, and S. V. Rykhlitskii, Tech. Phys. 58, 1529 (2013).

14. A. V. Sokolov, Optical Properties of Metals (GIFML, Moscow, 1961; American Elsevier, New York, 1967).

15. L. L. Gol'din, F. F. Igoshin, S. M. Kozel, and V. V. Mozhaev, Physics Labs (Nauka, Moscow, 1983) [in Russian].

16. N. N. Kosyrev, V. N. Zabluda, I. A. Tarasov, S. A. Lyaschenko, D. V. Shevtsov, S. N. Varnakov, and S. G. Ovchinnikov, RF Patent No. 2560148 (2015).
17. L. A. Golovan, V. Yu. Timoshenko, and P. K. Kashkarov, Phys. Usp. 50, 595 (2007).

18. I. H. Malitson, J. Opt. Soc. Am. 55, 1205 (1965).

19. W. Kohn and L. J. Sham, Phys. Rev. A 140, 1133 (1965).

20. G. Kresse and J. Furthmüller, Phys. Rev. B 54, 11169 (1996).

21. J. P. Perdew, K. Burke, and M. Ernzerhof, Phys. Rev. Lett. 77, 3865 (1996).

22. P. E. Blochl, Phys. Rev. B 50, 17953 (1994).

23. H. J. Monkhorst and J. D. Pack, Phys. Rev. B 13, 5188 (1976).

24. M. Gajdoš, K. Hummer, G. Kresse, J. Furthmüller, and F. Bechstedt, Phys. Rev. B 73, 045112 (2006).

25. P. B. Johnson and R. W. Christy, Phys. Rev. B 9, 5056 (1974).

26. M. R. Querry, Contractor Report CRDC-CR-85034 (1985).

27. M. Cazzaniga, L. Caramella, N. Manini, and G. Onida, Phys. Rev. B 82, 035104 (2010).

28. A. Zh. Brodskii and M. I. Urbakh, Sov. Phys. Usp. 25, 810 (1982).

Translated by $R$. Tyapaev 